\title{
ON THE NONEXISTENCE
}

\section{OF UNIMODULAR FUNCTIONS IN $R^{2}(X, d x d y)$}

\author{
ALFRED G. BRANDSTEIN
}

ABSTRACT. It is shown for certain planar sets, e.g., Brennan sets, with no interior, that if $f \in R^{2}(X, d x d y)$ and $|f|=1$ a.e. $d x d y$ then $f \equiv$ constant.

Introduction. Recently, invariant subspaces for $R^{2}(X, U)$ have been shown to exist by the method of constructing functions, $f$, such that $f \epsilon$ $R^{2}(X, U),|f|=1$ a.e. $d U$ and $f \not \equiv$ constant [1], [2], [3]. The question then arises if this method can be used for the general planar set with no interior and an arbitrary measure. We answer this question in the negative.

Main result. Let $X$ be a compact subset of the complex plane $\mathbf{C}$ and $u$ a finite positive Borel measure on $X$.

Definition 1. $R_{0}(X)$ is the class of rational functions which have no pole on $X$.

Definition 2. $R(X)$ is the uniform closure of $R_{0}(X)$ on $X$.

Definition 3. $R^{2}(X, u)$ is the closure of $R_{0}(X)$ in $L^{2}(X, u)$.

Theorem. There exists a planar set $X$ with no interior and having the property that if $f \in R^{2}(X, d x d y)$ and $f=1$ a.e. $d x d y$ then $f \equiv$ constant.

Lemma 1. Let $X$ be a compact subset of $\mathrm{C}$, and $m$ a finite positive measure on $X$. Suppose there exists a subset $Y$ of $X$ with $m(Y)>0$ such that for all $y \in Y, y$ is a bounded point evaluation on $R^{2}(X, m)$. If $\phi \epsilon$ $R^{2}(X, m)$ and $|\phi|=1$ a.e. $d m$, on $X$, then $\phi$ is constant on a set of positive m-measure.

Proof. By hypothesis there exists for every $y \in Y$, a $K y \in L^{2}(m)$ such that

$$
f(y)=\int f(X) K y(X) d m \text { for every } f \in R_{0}(X)
$$

\section{Define}

Received by the editors May 14, 1973.

AMS (MOS) subject classifications (1970). Primary 46J 10, 46J 20.

Key words and phrases. Bounded point evaluation, Brennan set. 


$$
\hat{f}(y)=\int f(X) K y(X) d m \text { for every } f \in R^{2}(m) \cap L^{\infty}(m)=R^{\infty} .
$$

We claim $f \rightarrow f(y)$ is multiplicative on $R^{\infty}$. For, let $f \in R_{0}(X), g \in R^{\infty}$. Then there exists a sequence $\left[g_{n}\right]$ in $R(X)$ with $g_{n} \rightarrow g$ in $L^{2}(m)$. Now

$$
f(y) g_{n}(y)=\left(f g_{n}\right)(y)=\int f g_{n} K y d m \rightarrow \int f g K y d m
$$

while $f(y) \cdot g_{n}(y) \rightarrow f(y) \cdot g(y)$. Thus

$$
f(y) g(y)=\int f g K y d m \quad \text { for } f \in R_{0}(X), \quad g \in R^{\infty} \text {. }
$$

Let now $F, G \in R^{\infty}$. Then

$$
(\widehat{F G})(y)=\int F G K y d m=\lim \int F_{n} G K y d m,
$$

where $F_{n} \in R_{0}(X), F_{n} \rightarrow F=\lim F_{n}(y) \hat{G}(y)=\hat{F}(y) \hat{G}(y)$ proving the claim.

Proposition 1 [Hoffman-Rossi]. Let $A$ be a subalgebra of $L^{\infty}(m)$ and let $\phi$ be a linear functional which is multiplicative on $A$ and which is weak ${ }^{*}$ continuous $i . e .$, there exists a function $F \in L^{1}(m)$ such that $\phi(f)=$ $\int f F d m$ for every $f \in A$. Then there is a nonnegative $k \in L^{1}(m)$ such that $\phi(f)=\int f k d m$ [4, pp. 460-461].

So by Proposition 1 there exists a $k y \in L^{1}(m)$ with $k y \geq 0$ such that

$$
\hat{f}(y)=\int f k y d m \text { for every } f \in R^{\infty} .
$$

Suppose now there exists a $\phi \in R^{2}$ with $|\phi|=1$ a.e. $d m$. Choose $\left[\phi_{n}\right], \phi_{m} \in R(X)$, with $\phi_{n} \rightarrow \phi$ in $L^{2}(m)$. Now by choosing a subsequence again denoted $\left[\phi_{n}\right]$, we have $\phi_{n}(y) \rightarrow \phi(y)$ a.e. $d m$. We conclude that for almost all $y \in Y, \hat{\phi}(y)=\phi(y)$ and so

$$
\phi(y)=\int \phi K y d m=\int \phi k y d m \text { by (4). }
$$

Therefore, for some $y \in Y,\left|\int \phi k y d m\right|=|\phi(y)|=1$. Without loss of generality we may assume $\int \phi k y d m=1$. As $k y>0$ and $|\phi|=1$ a.e. $d m$ it follows that $\phi k y \geq 0$ a.e. $d m$. Therefore, $\phi=1$ a.e. $d m$ on the set $[z: k y(z) \neq 0]$, and this set has positive $m$-measure. Q.E.D.

Proposition 2 [Brennan]. There exists a compact subset on the plane with no interior such that every function constant on a set of positive $-d x d y$ 
measure is constant a.e. on $X$; moreover there is a bounded point evaluation at almost every point of X L3, p. 3].

Proof. Apply Lemma 1 to Brennan's set. Q.E.D.

\section{REFERENCES}

1. A. G. Brandstein, Function spaces related to hypo-Dirichlet algebras, Doctoral Dissertation, Brown University, Providence, R. I., 1972.

2. J. Brennan, Invariant subspaces and rational approximation, J. Functional - Analysis 7 (1971), 285-310.

3. - Approximation in the mean and quasi-analyticity, University of Kentucky, Math. Dept., 1972.

4. K. Hoffman and H. Rossi, Extensions of positive weak*-continuous functions, Duke Math. J. 34 (1967), 453-466. MR 37 \#763.

U.S.A. HARRY DIAMOND LABORATORIES, ADELPHI, MARYLAND 20783 